\title{
量子通用包络代数的非齐性实现与 谱部分代数化方法的推广*
}

\author{
孙昌 渼 \\ (东北师范大学物理系, 长春 130024 ; 南开数学矿究所理论物理矿究室, 天津 300071 )
}

\section{关键词量子通用包络代数、玻色子实现、谱代数化}

目前, 由于联系于杨-Baxter 方程的非线性物理的炒展, 量子群和量子通用包络代数 (QUEA) 及其表示理论已成为数学物理的重要研究领域 ${ }^{[1,2]}$. 最近, 为了明显地构造 QUEA 的表示, 几位作者独立地建议了 QUEA 的 $q$-变形玻色子(振子) 实现 ${ }^{[3-5]}$. 其中, 我们的工作 ${ }^{[4]}$ 不仅涉及到 $S U(2)$ 情况, 而且着重讨论了 $S U(n)(n>2)$ 情况.

本文将由一般代数观点构造出量子通用包络代数 $\mathscr{U}{ }_{q}(S U(2))$ 的非齐性 $q$-变形微分实 现, 并给出其积分表示. 由此, 我们把最近发展的量子力学谱部分代数化方法 ${ }^{[6,7]}$ 推广一到量子群 (或 QUEA) 情形, 从而得到一类非定域微分方程 (或微分-积分方程) 部分谱的精确解. 当 $q \rightarrow 1$ 时, 此类问题恰好描述磁场中的自旋进动. 本文讨论只涉及 $q^{p} \neq 1(\forall p \in \mathbf{N}=\{1$, $2, \cdots\})$ 的 $S U(2)$ 情形, 但本文讨论可推广到 $S U(n)$ 和 $q^{p}=1(P \in \mathbb{N})$ 情况.

\section{一、从齐性玻色子实现到非齐性微分实环}

根据文献 [4],由满足

$$
\begin{gathered}
a_{i} a_{j}^{+}=\left\{\begin{array}{l}
a_{j}^{+} a_{i}, j \neq i, \\
q^{-1} a_{i}^{+} a_{i}+q^{N_{i}}, j=i, q \in \text { 复数域 } \mathrm{C} ;
\end{array}\right. \\
{\left[N_{i} a_{j}^{ \pm}\right]= \pm \delta_{i} a_{j}^{+},\left[a_{i}^{ \pm}, a_{j}^{ \pm}\right]=\left[N_{i}, N_{i}\right]=0, i, j=1,2}
\end{gathered}
$$

的 $q$-变形玻色子算子 $a_{i} \equiv a_{i}^{-}, a_{i}^{+}$以及 $N_{i}(i=1,2)$, 可以构造 $\mathscr{U}_{q}(S U(2))$ 的 $q$-变形齐 性玻色子实现:

$$
J_{+}=a_{1}^{+} a_{2}, J_{-}=a_{2}^{+} a_{1}, J_{3}=N_{1}-N_{2} .
$$

它在 $q$-变形 Fock 空间 $\left.\mathscr{F}_{q}:\left\{\left|n_{1}, n_{2}\right\rangle=a_{1}^{+n_{1}} a_{2}^{+n_{2}}|0\rangle\left|n_{i} \in \mathrm{N}, a_{i}\right| 0\right\rangle=N_{i}|0\rangle=0, i=1,2\right\}$ 定义了 $\mathscr{U}{ }_{a}(S U(2))$ 的一个表示. 而在 $\mathscr{F}_{a}$ 的不变子空间 $V^{[N]}(N \in \mathrm{N}) ;\left\{F_{N}(n)=\mid n\right.$, $N-n\rangle \mid n=0,1,2, \cdots, N\}$ 上, 我们则得到一个 $N+1$ 有限维表示 $\Gamma^{[N]}$ :

$$
\left\{\begin{array}{l}
J_{+} F_{N}(n)=[N-n] F_{N}(n+1), \\
J_{-} F_{N}(n)=[n] F_{N}(n-1), \\
J_{3} F_{N}(n)=\left(n-\frac{N}{2}\right) F_{N}(n),
\end{array}\right.
$$

本文 1990 年 9 月 13 日收到.

* 国家青年自然科学基金资助课题. 
其中我们对任何算符或 C 数 $f$ 定义了 $[f]=\frac{q^{\prime}-q^{-1}}{q-q^{-1}}$.

现在考虑由 $V^{: N]}$ 到 Bargmann 空间 $\mathscr{B}:\{f(z) \mid f$ 是 $\mathrm{C}$ 上的解析函数 $\}$ 的 $1-1$ 映射 $\Phi$ : $F_{N}(n) \rightarrow Z^{*}$, 则可交换胦射图

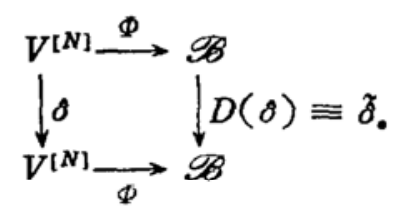

对任何 $\theta-J_{ \pm}, J_{3}$ 成立确定了 $\theta=J_{ \pm}, J_{3}$ 的 $q$-变形微分实现 $D(\theta)=\tilde{\delta}=\tilde{J}_{ \pm}, \tilde{J}_{3}$ :

$$
\left\{\begin{array}{l}
\tilde{J}_{+}=z \cdot\left[N-z \frac{d}{d z}\right], \\
\tilde{J}_{-}=(1 / z)\left[z \frac{d}{d z}\right] \equiv D_{x}, \\
\tilde{J}_{3}=z \frac{d}{d z}-N / 2
\end{array}\right.
$$

其中当 $q \rightarrow 1, D_{z} \rightarrow \frac{d}{d z}$, 故称 $D_{x}$ 为 $q$ 变形微分算符. 若把(3)式中的 $z$ 和 $\frac{d}{d z}$ 分别换成普 通的玻色子算子 $b^{+}$和 $b\left(\left[b, b^{+}\right]=1\right)$, 则(3)式给出 $\mathscr{U}{ }_{q}(S U(2))$ 非齐性玻色子实现. 因 此, 我们称 (3)式为 $\mathscr{U}{ }_{q}(S U(2))$ 非齐性 $q$-变形微分实现. 直接计算可验证(3)式满足

$$
\left[\tilde{J}_{+}, \tilde{J}_{-}\right]=\left[2 \tilde{J}_{3}\right],\left[\tilde{J}_{3}, \tilde{J}_{ \pm}\right]= \pm \tilde{J}_{ \pm} .
$$

\section{二、作为积分算子的 $\tilde{J}_{ \pm}$}

现在我们证明: $\mathscr{U}_{a}(S U(2))$ 非齐性实现(3)式中的 $\tilde{J}_{ \pm}$或 $D_{s}$ 和 $\left[N-z \frac{d}{d z}\right]$ 是 $\mathscr{B}$ 上的 积分算子. 事实上,对任何解析函数 $f(z) \in \mathscr{B}$ 有

$$
f(z)=\sum_{n=0}^{\infty} C_{n} \cdot Z^{n}, C_{z} \in \mathrm{C},
$$

且

$$
\begin{aligned}
& D_{z}\left(z^{n}\right)=[n] z^{n-1}, \\
& {\left[N-z \frac{d}{d z}\right]\left(z^{n}\right)=[N-n] z^{n}}
\end{aligned}
$$

从而有

$$
\begin{gathered}
D_{s} f(z)=\frac{f(q z)-f\left(q^{-1} z\right)}{z\left(q-q^{-1}\right)}, \\
{\left[N-z \frac{d}{d z}\right] f(z)=\frac{q^{N} f\left(q^{-1} z\right)-q^{-N} f(q z)}{q-q^{-1}},}
\end{gathered}
$$

其中 $(5 a)$ 已先后在文献 $[8,9]$ 中给出. 利用柯西公式及 $(5 a, b)$, 不难写出 $J_{ \pm}$的积分实现

$$
\begin{aligned}
& \tilde{J}_{-} f(z)=\frac{1}{2 \pi i} \oint_{\epsilon} A(\xi, z) f(\xi) d \xi, \\
& \tilde{J}_{+} f(z)=\frac{1}{2 \pi i} \oint_{0} B(\xi, z) f(\xi) d \xi,
\end{aligned}
$$


其中 $c$ 包围 $q^{-1} z$ 和 $q z$ 点的围道, 而

是积分核.

$$
\begin{aligned}
& A(\xi, z)=\frac{1}{(\xi-q z)\left(\xi-q^{-1} z\right)}, \\
& B(\xi, z)=\frac{z([N] \xi-[N+1] z)}{(\xi-q z)\left(\xi-q^{-1} z\right)}
\end{aligned}
$$

\section{三、谱部分代数化方法的量子群推广}

由 Turbiner 和 Shifman 发展的量子力学谱部分代数化方法是原来的谱生成 代数理 论 ${ }^{[10]}$ 的推广. 它的基本思想是: 利用李代数的非齐次微分实现（它的一般计算方法在文献 [11]中给出), 把量子体系的 Hamiltonian $\hat{H}=\hat{H}\left(\cdots-i \hbar \frac{d}{d x} \cdots\right)$ 化为李代数 $\mathscr{L}$ (基为 $T_{a}$, $a=1,2, \cdots, l)$ 通用包络代数 $\mathscr{U}(\mathscr{L})$ 的元素, 即 $\hat{H}=\hat{H}\left[T_{1}, T_{2}, \cdots, T_{l}\right]$. 这时, 我们选择 $\mathscr{L}$ 的 $d$ 维不可约表示 $\Gamma^{[A]}$ 的基作为 Hilbert 空间基的一部分并在此基上写出 $\hat{H}$ 的矩阵, 则 $\hat{H}$ 化为准对角型, 即 $\hat{H}=\operatorname{diag}\left(H^{[A]}, H^{\prime}\right)$, 其中 $H^{[A]}$ 是 $d \times d$ 矩阵; 而 $H^{\prime}$ 是一个无穷维或 维数很高的矩阵. 这时我们可以在不影响 $H^{\prime}$ 的前提下对角化 $H^{[\Lambda]}$ 从而得到 $\hat{H}$ 的部分能谱.

我们的推广是: 把通用包络代数 $\mathscr{U}(\mathscr{S})$ 换成它所对应的量子通用包络代数 $\mathscr{U}{ }_{q}(\mathscr{L})$, 而哈密顿量 $\hat{H}_{q}$ 作为 $\mathscr{U}_{q}(\mathscr{L})$ 元素可写成 $\mathscr{U}_{q}(\mathscr{L})$ 生成元 $q$-变形非齐次微分实现的函 数, 即 $\hat{H}_{q}=\hat{H}\left[\widetilde{T}_{1}, \widetilde{T}_{2} \cdots \widetilde{T}_{l}\right]$. 这样我们可以在 $\mathscr{U}{ }_{q}(\mathscr{L})$ 的不可约表示空间上部分地对角 化出 $\hat{H}$ 的谱, 即求解 $\hat{H}_{q} \psi=\lambda \Psi$. 现在以 $S U(2)$ 情况为例, 令

$$
\hat{H}_{q}=\alpha \tilde{J}_{+}+\alpha * \tilde{J}_{-}+\beta J_{3} \quad(\alpha \in \mathrm{C}, \beta \text { 为实参锌 }) \text {. }
$$

利用 $\tilde{J}_{ \pm}$和 $\tilde{J}_{3}$ 的微分 (积分) 实现 (3) 和 (6)式, 本征方程 $\hat{H}_{q} \Psi(z)=\lambda \Psi(z)$ 可写为非定域微分 方程

$$
\begin{gathered}
\left(\alpha z^{2} q^{N}-\alpha^{*}\right) \Psi\left(q^{-1} z\right)-\left(\alpha z^{2} q^{-N}-\alpha^{*}\right) \Psi(q z)+\beta z^{2}\left(q-q^{-1}\right) \frac{d}{d z} \Psi(x) \\
=\left(\lambda+\beta \frac{N}{2}\right)\left(q-q^{-1}\right) z \Psi(z),
\end{gathered}
$$

或积分-微分方程

$$
\begin{gathered}
\frac{1}{2 \pi i} \oint_{c} K(\xi, z) \Psi(\xi) d \xi+\beta z \frac{d}{d z} \Psi(z)=\left(\lambda+\beta \frac{N}{2}\right) \Psi(z), \\
K(\xi, z)=\alpha A(\xi, z)+\alpha^{*} A(\xi, z) .
\end{gathered}
$$

现在我们完全可以用代数的方法求解(8)或(9)式,为此我们定义 $\mathscr{B}$ 的子空间 $B^{[i]}$ :

$$
\left\{\begin{array}{l}
|j, m\rangle=\prod_{k=m}^{-(j-1)}\left(\frac{[j+m+1]}{[j-m]}\right)^{\frac{1}{2}} z^{j+m}, j=\frac{N}{2}, m=j, j-1, \cdots,-j+1, \\
|j,-j\rangle=1,
\end{array}\right.
$$

则 $B^{[j]}$ 荷载着 $\mathscr{U}_{q}(S U(z))$ 的不可约表示 $D^{[i]}:^{[2-5]]}$

$$
\left\{\begin{array}{l}
\tilde{J}_{ \pm}|j, m\rangle=([j \mp m][j \pm m+1])^{\frac{1}{2}}|j, m \pm 1\rangle, \\
\bar{J}_{3}|j m\rangle=m|j, m\rangle,
\end{array}\right.
$$

在 $B=\Sigma \oplus B^{[i]}$ 上, $\hat{H}_{q}=\operatorname{diag}\left(H_{q}^{\left[\frac{1}{2}\right]}, H_{q}^{[1]}, \cdots, H_{q}^{[j]}, \cdots\right)$ 其中对角块 $H_{q}^{[i]}$ 是 $(2 j+1) \times(2 j+1)$ 矩阵, 我们可以在不影响其它对角块的前提下, 把 $H_{q}^{[i]}$ 对角化. 例如, $j=1$ 时,

1306 


$$
H_{a}^{\prime \prime \prime}=\left(\left\langle 1, m\left|\hat{H}_{q}\right| 1, m\right\rangle\right)=\left[\begin{array}{ccc}
\beta, & \sqrt{[2]} \alpha & 0 \\
\sqrt{[2]} \alpha^{*} & 0 & \sqrt{[2] \alpha} \\
0 & \sqrt{[2]} \alpha^{*} & -\beta
\end{array}\right],
$$

对角化 (11) 式,不难求得方程 (8)和(9)的一组解:

$$
\begin{gathered}
\lambda_{m}=m\left[z\left(q+q^{-1}\right)|\alpha|^{2}+\beta^{2}\right]^{\frac{1}{2}}, m=0, \pm 1, \\
\mathbb{P}_{m}(\boldsymbol{z})-\frac{\left(q+q^{-1}\right)^{\frac{1}{2}} \alpha}{\lambda_{m}-\beta}|1,1\rangle+\frac{\left(q+q^{-1}\right)^{\frac{1}{2}} \alpha^{*}}{\lambda_{m}+\beta}|1-1\rangle+|1,0\rangle .
\end{gathered}
$$

值得指出的是, 当 $q \rightarrow 1, \hat{H}_{q} \rightarrow \hat{H}-\boldsymbol{B} \cdot \boldsymbol{S}$, 这洽是磁场中自旋进动的哈密顿量, 其中 $S-J_{a_{a \rightarrow 1}}$ 是普通角动量算符, $B_{x}-\alpha+\alpha^{*}, B_{y}=i\left(\alpha-\alpha^{*}\right), B_{z}=\beta$.

致的: 感谢郭汉英、葛墨林以及吴兆颜教授的讨论和鼓励.

\section{参考文献}

[1] Drinfeld, V.G., Proc. ICM Berkely, 1986, 798.

[2] Jimbo, M., Lett. Math. Phys., 11(1986), 247.

[3] Beidenharn, L.C., J. Phys. A, 22(1989), L873.

[4] Sun, C.P. and Fu, H.C., J. Phys. A, 22(1989), L983.

[5] Macfarlane, A.J., J. Phys. A, 22 (1989). 4581.

16] Turbiner, A.V., Comm. Math. Phys., 118 (1988). 467.

[7] Shifman, M., Inter. J. Mod. Phys. A, 4(1989), 2897.

「8] Alveraz-Gaume, L. et al., Nucl. Phys., B330 (1990), 435.

[9] Sun, C.P. and Fu, H.C., Commun. Theor. Phys., 13 (1990), 217.

[10] Wybourne, B.G., Classical Groups for Physicirts, John Wiley \& Sons, New York, 1974. 207.

[i1] Fu. H.C. and Sun, C.P., J. Math. Phys., 31(1990), 287; 2797. 